\title{
To head or to heed? Beyond the surface of selective action inhibition: a review
}

\section{Wery P.M. van den Wildenberg ${ }^{1 *}$, Scott A. Wylie ${ }^{2}$, Birte U. Forstmann ${ }^{3}$, Borís Burle $^{4}$, Thierry Hasbroucq $^{4}$ and K. Richard Ridderinkhof ${ }^{1,3}$}

\author{
'Department of Psychology, Amsterdam Center for the Study of Adaptive Control in Brain and Behavior, University of Amsterdam, Amsterdam, Netherlands \\ 2 Neurology Department, University of Virginia Health Systems, Charlottesville, VA, USA \\ ${ }^{3}$ Spinoza Center for Neuroimaging, University of Amsterdam, Amsterdam, Netherlands \\ ${ }^{4}$ Laboratoire de Neurobiologie de la Cognition, Centre National de la Recherche Scientifique, Aix-Marseille Université, Marseille, France
}

Edited by:

Michael X. Cohen, University of

Amsterdam, Netherlands

Reviewed by:

Tobias Egner, Duke University, USA

Birgit Stürmer, Humboldt Universität

Berlin, Germany

${ }^{*}$ Correspondence:

Wery P.M. van den Wildenberg,

Department of Psychology, University

of Amsterdam, Roetersstraat 15, 1018

WB Amsterdam, Netherlands.

e-mail: w.p.m.vandenwildenberg@uva.nl
To head rather than heed to temptations is easier said than done. Since tempting actions are often contextually inappropriate, selective suppression is invoked to inhibit such actions. Thus far, laboratory tasks have not been very successful in highlighting these processes. We suggest that this is for three reasons. First, it is important to dissociate between an early susceptibility to making stimulus-driven impulsive but erroneous actions, and the subsequent selective suppression of these impulses that facilitates the selection of the correct action. Second, studies have focused on mean or median reaction times (RT), which conceals the temporal dynamics of action control. Third, studies have focused on group means, while considering individual differences as a source of error variance. Here, we present an overview of recent behavioral and imaging studies that overcame these limitations by analyzing RT distributions. As will become clear, this approach has revealed variations in inhibitory control over impulsive actions as a function of task instructions, conflict probability, and between-trial adjustments (following conflict or following an error trial) that are hidden if mean RTs are analyzed. Next, we discuss a selection of behavioral as well as imaging studies to illustrate that individual differences are meaningful and help understand selective suppression during action selection within samples of young and healthy individuals, but also within clinical samples of patients diagnosed with attention deficit/hyperactivity disorder or Parkinson's disease.

Keywords: action control, response inhibition, prefrontal cortex, basal ganglia, interference control

\section{IMPULSIVE ACTIVATION AND SELECTIVE SUPPRESSION OF ACTIONS DURING CONFLICT}

"Look before you leap" and "haste makes waste" are just two examples of every-day expressions - not to say clichés - that point to the precarious balance between reacting impulsively on the one hand versus carefully weighing your response options before acting. The need for behavioral restraint during decision making is particularly relevant when confronted with irrelevant attributes or changes in the environment that activate unintentional response tendencies that conflict with the desired appropriate behavior. This review first introduces a theoretical framework, the activation-suppression model that describes the effects of processing conflicting information on behavior. Importantly, the activation-suppression model generates two specific individual parameters that represent the temporal aspects of (1) the susceptibility to make fast impulsive reactions, and (2) the proficiency of selective inhibitory control over these unwanted actions to facilitate the selection of the appropriate response. This useful dissociation is illustrated by an overview of behavioral studies that helped identify experimental variables (e.g.,

Abbreviations: $\mathrm{AD} / \mathrm{HD}$, attention deficit/hyperactivity disorder; $\mathrm{CAF}$, conditional accuracy function; EEG, electroencephalography; EMG, electromyography; IFC, inferior frontal cortex; pre-SMA, pre-supplementary motor area; RT, reaction time; STN, subthalamic nucleus. conflict probability and task instructions) as well as differences between groups of individuals (e.g., age, and clinical diagnoses like attention deficit/hyperactivity disorder [AD/HD] and Parkinson's disease) that affect the initial activation and selective suppression of unwanted action tendencies. Finally, we present an overview of recent brain imaging studies that focus on the underlying neural mechanisms. As will become clear, activation and effective selective suppression of unwanted actions is expressed by subthreshold muscle activity that can be measured using electromyography (EMG) recorded from the incorrect response hand. Application of brain imaging techniques advanced our understanding of the cortical activation patterns, structural connectivity, and subcortical contributions that are associated with the voluntary selection of actions in the face of conflicting response impulses.

\section{PARADIGMS THAT INDUCE CONFLICT}

Over the years, several reaction time (RT) paradigms have been developed to study cognitive processing in conflicting situations in which competing response tendencies are simultaneously active. Widely used variants of such conflict paradigms are the Stroop task (Stroop, 1935) and the Eriksen flanker task (Eriksen and Eriksen, 1974), that all share a common cognitive processing architecture, the dual-route model (Kornblum et al., 1990; Kornblum, 1994). Although these different tasks clearly induce interference produced 
by response conflict, the source that triggers interference may vary across conflict tasks. For example, the Stroop interference involves a number of potential sources of variance, such as response conflict, perceptual conflict (as in the Flanker task), and semantic interference. Alternatively, the Simon task (Simon, 1967, 1990) induces response conflict and inherently avoids interference associated with perceptual or semantic conflict. The Simon task (Simon, 1967, 1990 ) in particular provides a context in which irrelevant stimulus information can elicit a strong response impulse that interferes with goal-directed action (see Figure 1). The task typically requires a fast button-press to a goal-relevant stimulus aspect embedded in a goal-irrelevant stimulus dimension. For example, participants may be instructed to issue a discriminative response according to the color of a stimulus; to press the left response button when the circle is blue and to issue a right-hand button press to a green circle. Notably, the colored circles are presented to the left or right of a fixation point.

According to dual-route models of information processing, the spatial location of the stimulus, despite being irrelevant, automatically and rapidly activates the spatially corresponding response via a direct processing route (Kornblum et al., 1990; de Jong et al., 1994; Eimer et al., 1995; Ridderinkhof, 2002a; Ridderinkhof et al., 2004c). In contrast, the relevant stimulus feature engages a deliberate processing route that utilizes a slower controlled translation of relevant stimulus features into a correct response according to task instructions. On compatible trials, the direct route and the deliberate processing route converge on activation of the same response and, in so doing, facilitate both RT and accuracy. In contrast, on incompatible trials, the two responses, that is, the one activated by the automatic processing of the circle's spatial location and the one activated according to the deliberate processing of the circle's color, are incompatible, thereby slowing RT and increasing error rates. The mean compatibility or interference effect is taken to reflect the extra demands and time required to overcome the interference caused by the incorrect response activation produced on incompatible trials that are absent in compatible trials due to

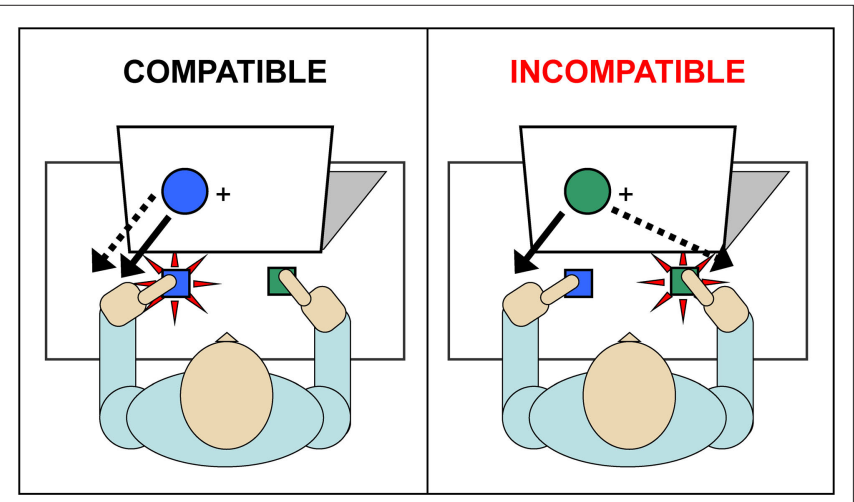

FIGURE 1 | Simon task. Participants press the left button to a blue circle and a right button to a green circle (dashed line). Responses are also driven by an irrelevant stimulus dimension, circle location, as indicated by the solid line. For compatible trials, both relevant (color) and irrelevant (location) stimulus dimensions activate the correct action. On incompatible trials, the irrelevant dimension activates an incorrect response tendency, which interferes with selection of the correct response. the facilitation from direct-route processing (Ridderinkhof et al., 2004c). However, as will become clear in the next section, essential information about the temporal aspects of information processing in conflict situations is lost if behavioral analyses are restricted to the mean interference effect.

\section{THE ACTIVATION-SUPPRESSION MODEL}

Interestingly, many Simon studies that compared fast versus slower responses reported a reduction of the Simon effect if RT is relatively long. These essential temporal dynamics are revealed if the Simon effect is computed as a function of response time, but easily missed if the overall mean Simon effect is taken as the dependent measure of interference control. The activation-suppression model (see Figure 2), a recent extension of the dual-route architecture, and a related analytic technique were developed to incorporate the temporal dynamics that underlie the expression of impulsive errors followed by a gradual build-up of selective suppression as an act of cognitive control (Ridderinkhof, 2002b; Ridderinkhof et al., 2004c). Based on these temporal aspects, the model predicts that faster reactions on conflict trials should be more vulnerable to impulsive actions that are captured by the irrelevant stimulus dimension. Conversely, the model asserts that slower reactions on incompatible trials are less likely to be negatively impacted by incorrect action impulses because selective suppression had time to accrue to counteract these involuntary impulses. Given that the suppression mechanism needs time to become effective, slower responses benefit more from the effect of selective suppression than faster responses. Therefore, slow responses show a relatively less pronounced interference effect. For example, in the Eriksen flanker task, the interference effect increases with RT, but to a lesser extent for slower RT segments compared to faster segments (Wylie et al., 2007, 2009a,b). In the Simon task, the interference effect reduces in absolute terms, and can even reverse (i.e., go below 0 ) over time. Here, the slowest RT segment is associated with the smallest Simon effect in absolute value.

Thus, the activation-suppression model refines dual-route models of interference effects by incorporating specific hypotheses about the temporal dynamics of incorrect response activation followed by top-down suppression of unwanted impulsive actions. As illustrated next, the time-courses of these dissociable processes are

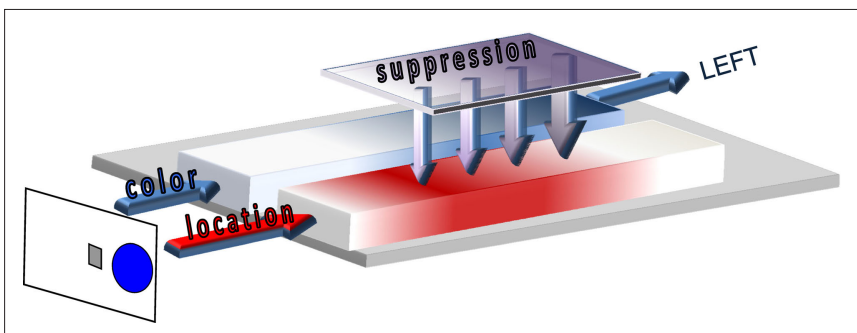

FIGURE 2 |Activation-suppression model. The relevant stimulus dimension (color blue) is processed by the slow deliberate route (represented in blue) while the irrelevant location dimension (right location activating the right hand) is processed by the fast direct route (in red). Selective suppression of the location-based activation by the inhibition module (represented in purple) needs time to build up, and facilitates the selection and execution of the correct left-hand response. 
masked using overall mean interference effects, but exposed using RT distributional analyses of fast errors and interference effects, respectively. These analyses empirically show that incompatible trials yield an early automatic response impulse that is distinct from a later controlled top-down response suppression mechanism.

\section{CONDITIONAL ACCURACY FUNCTIONS REVEAL IMPULSIVE RESPONSE ACTIVATION}

According to the activation-suppression model, the susceptibility to react impulsively is revealed by the relation between fast errors and response speed. Stronger initial impulsivity on incompatible trials is expressed by an increase in the proportion of fast errors as less time is available for the build-up of suppression to counter this incorrect activation (Kornblum et al., 1990). Thus, plotting accuracy rates for incompatible trials as a function of RT, using the conditional accuracy function or CAF, provides a means for studying the strength of automatic responses that are captured in conflicting situations, with stronger capture associated with a higher frequency of fast response errors.

Notably, the strength of automatic response capture is sensitive to various experimental factors that directly affect conflict processing. Wylie et al. (2009b) used an Eriksen flanker paradigm that required participants to respond to a central arrow stimulus while ignoring flanking arrow distracters. These flanking arrows signaled either the same (i.e., are compatible $\rightarrow \rightarrow \rightarrow \rightarrow \rightarrow$ ) or signaled the opposite response as the target arrow (i.e., are incompatible $\rightarrow \rightarrow \leftrightarrows \rightarrow \rightarrow$. Responses are typically slowed and less accurate when the flanking arrows point to the opposite direction as the central arrow, thus inducing conflict. Participants performed the Flanker task under instructions that either emphasized speed or accuracy of responses. Distributional analyses were used to investigate the temporal dynamics of direct response activation on conflict trials on which target and flankers signal opposite responses. According to the activation-suppression hypothesis, the proportion of fast errors reflects the strength of initial response capture by the incompatible flankers, quantified by the slope of the first segment of the CAF connecting the first two points (see Figure 3). Steeper CAF slopes reflect a higher proportion of fast errors, suggestive of stronger initial activation of the incorrect response (Ridderinkhof, 2002b; Wylie et al., 2009b). As can be seen in Figure 3, responses on compatible trials are associated with near perfect accuracy. In contrast, incompatible flanker trials produced significantly more errors (i.e., steeper positive-going slope) in the fast proportion of the RT distribution. Interestingly, the effect of flanker incompatibility on making impulsive errors was modified by task instructions. An emphasis on the importance of response speed greatly increased errors on conflict trials compared to the instruction condition that stressed accuracy. This was especially true for the fast proportion of responses on incompatible trials (Band et al., 2003; Wylie et al., 2009b).

The speed-accuracy study by Wylie et al. (2009b) showed that response strategies affect the susceptibility to making fast impulsive errors in conflict situations. In addition to these macro-adjustments that involve long-term modifications in speed-accuracy strategy, CAFs also revealed micro-adjustments on a trial-by-trial level. These between-trial adjustments in interference control on trial $\mathrm{N}$ are triggered by incompatibility on trial $\mathrm{N}-1$. Ridderinkhof (2002b) administered a version of the Simon task to a sample of healthy participants and focused on accuracy rates on trials that were preceded either by a correct response or by a response error. Between-trial adjustments were evidenced by an increase in accuracy for fast responses from chance level on post-correct trials to about $75 \%$ on trials that followed an error. This increase in accuracy on post-error trials relative to post-correct trials might be related to a top-down controlled shift in speed-accuracy trade-off because of post-error slowing. Another example of between-trial control is presented by Stins et al. (2007) who also plotted accuracy as a function of response speed. They observed that the accuracy of fast responses to incompatible flanker trials that were preceded by compatible trials was as low as $26 \%$, which is well below chance

\section{A Speed Instructions}

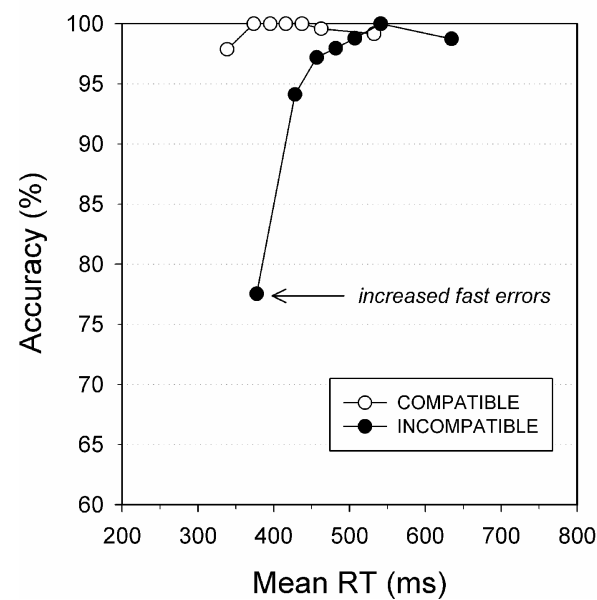

B

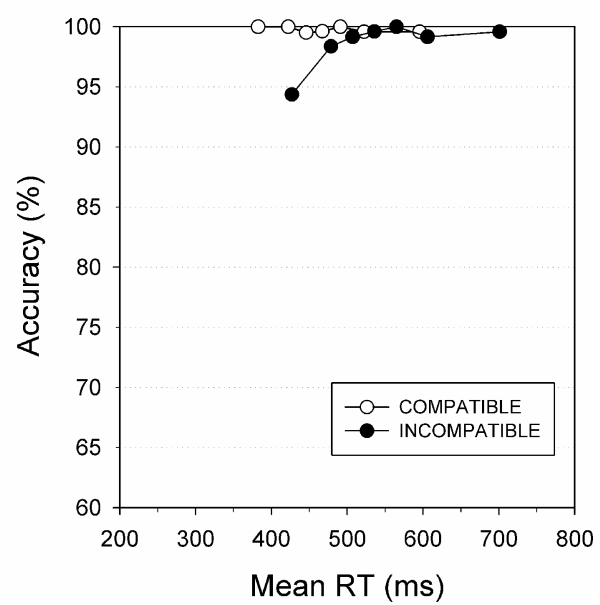

FIGURE 3 | Task instructions affect fast errors. Conditional accuracy functions illustrate the increased tendency to commit fast errors to incompatible flanker (black circles) compared to compatible flanker trials (white circles). The proportion of fast errors on conflict trials increases under instructions that emphasize speed (A) compared to accuracy of responding (B). Figure modified after Wylie et al. (2009b). 
level. In contrast, accuracy for fast incompatible trials following incompatible trials increased to up to 50\%. Wylie et al. (2010) confirmed that this between-trial conflict adaptation is preserved in individuals diagnosed with Parkinson's disease. Figure 4 illustrates that healthy participants as well as Parkinson's patients performing on a Simon conflict task essentially show a comparable pattern of reduced direct response activation on incompatible trials following an incompatible trial (i.e., after a conflict situation, Figure 4B) compared to incompatible trials preceded by a compatible trial (Figure 4A).

That same clinical study also revealed individual differences in the susceptibility to making fast impulsive errors within the group of Parkinson's patients (Wylie et al., 2010). Forty-five patients with mild to moderate motor symptoms performed the Simon task and were divided into three subgroups that reflected relatively less severe, moderately severe, and most severe motor symptoms according to a clinical motor rating scale. CAFs were computed to study impulsive errors on incompatible trials. Group analyses revealed that patients with the most severe motor symptoms committed a significantly higher proportion of fast impulsive errors compared to the two subgroups with less severe motor symptoms. Thus, the activation of unwanted response impulses, as measured by CAFs in the Simon task, is sensitive to individual as well as group differences in the susceptibility to impulsive action selection (Wylie et al., 2010).

The above studies illustrate the usefulness of distributional analyses to reveal a pattern of within- and between-trial adjustments that affect the susceptibility of making fast impulsive errors that are driven by irrelevant information. Note that analyses of overall accuracy performance do not reveal these temporal effects on behavior. The next section provides an overview of studies that used distributional analyses to quantify the temporal aspects of selective suppression to counteract the activation of unwanted response tendencies in an attempt to resolve response conflict.
DELTA PLOTS REVEAL THE DYNAMICS OF SELECTIVE ACTION INHIBITION A delta plot is a graphical representation that displays the temporal dynamics of the RT difference between two experimental conditions (the delta value) as a function of response speed (see Figure 5A; (de Jong et al., 1994; Ridderinkhof, 2002a,b). Take two hypothetical experimental conditions, $\mathrm{X}$ and $\mathrm{Y}$, where one condition is associated with slower responses than the other. The typical pattern is that the proportional difference in RT between the two conditions is similar across RT segments (cf. Luce, 1986). Consequently, the absolute RT difference between conditions $\mathrm{X}$ and $\mathrm{Y}$ increases from fast to slower segments and the slope values of the lines connecting the delta values are positive going (see Ridderinkhof et al., 2005; Wagenmakers et al., 2005; Speckman et al., 2008). If the two RT distributions belong to the same family, the delta plot should be linear. In contrast, conflict paradigms like the Eriksen flanker task and the Simon task typically yield a different delta-plot pattern. Here, delta slopes in the slower RT segments tend to level off or even go negative, indicating that the differences between response latencies in the $\mathrm{X}$ and $\mathrm{Y}$ conditions decrease instead of increase as a function of RT.

The activation-suppression hypothesis postulates that this leveling-off is indicative of selective suppression by a top-down control mechanism. This selective suppression acts to resolve response interference by counteracting the initial (incorrect) response activation that is activated by the direct processing route. In contrast to the rapid engagement of the response capture mechanism, topdown suppression takes time to build-up and, therefore, is most effective for responses that are relatively slow (Burle et al., 2002; Ridderinkhof, 2002a; Ridderinkhof et al., 2004c; Forstmann et al., 2008a,b; Wylie et al., 2010). For instance, the faster one responds, the less likely it is that suppression will have accrued to a level that is sufficient to counteract response capture. Rather, slower responses are more likely to benefit from the build-up of suppression to resolve interference. Consequently, correct slow responses to compatible

\section{A Preceding Trial is Compatible}

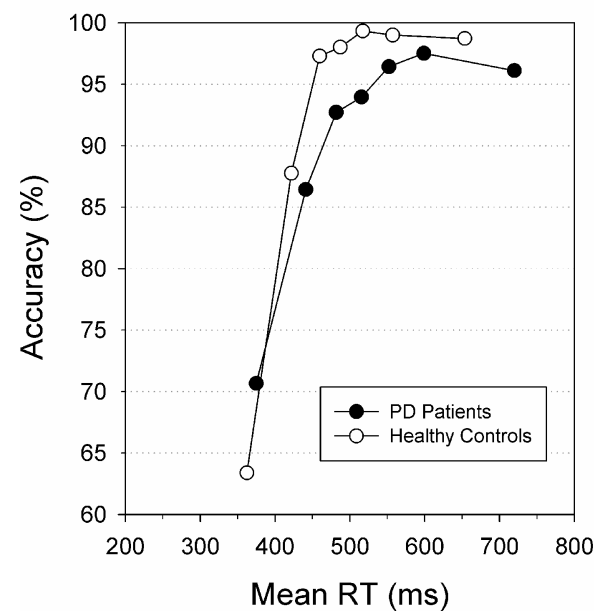

FIGURE 4 | Effect of preceding trial on fast errors. Conditional accuracy functions for incompatible trials preceded by compatible trials (A) or by incompatible trials (B) for individuals diagnosed with Parkinson's disease (black circles) and healthy controls (white circles). For both

\section{B Preceding Trial is Incompatible}

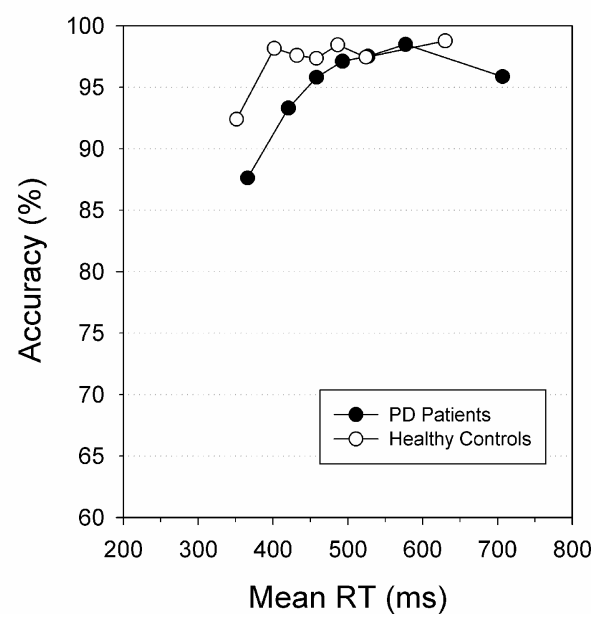

groups, fast responses on incompatible trials are associated with increased error rates, notably on trials that follow compatible trials. Both groups displayed these between-trial adjustments. Figure modified after Wylie et al. (2010). 


\section{A RT Delta Plot}

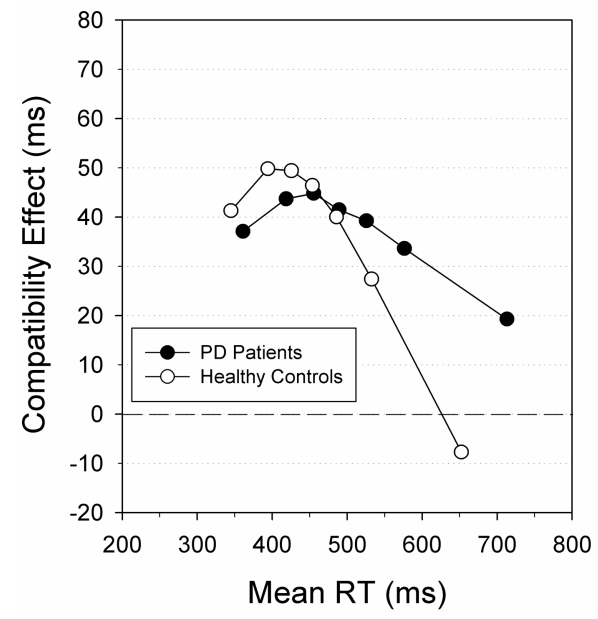

B

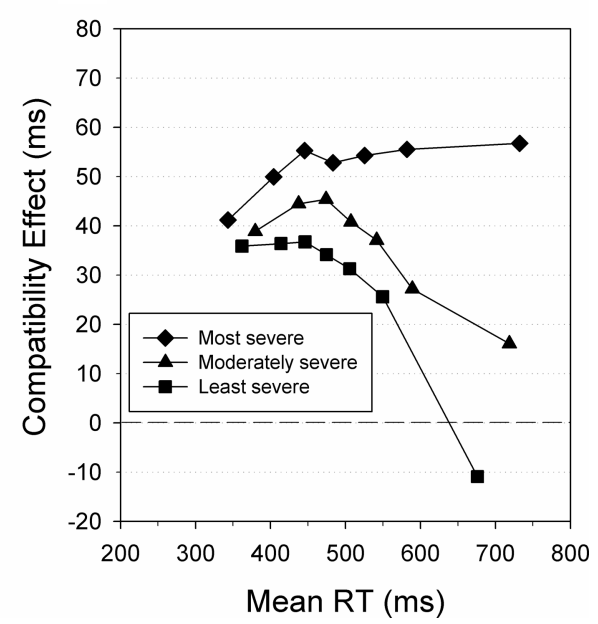

FIGURE 5 | Delta plot for RT. (A) Delta plot illustrating impaired selective suppression in patients diagnosed with Parkinson's disease (black) compared to age-matched healthy controls (white). (B) Delta plot illustrating impaired selective suppression in mild to moderate PD patients associated with relatively more severe clinical symptoms compared to patients with less severe symptoms. Figure modified after Wylie et al. (2010).

stimuli will be less facilitated by position-driven response capture, whereas correct slow responses to incompatible stimuli will be less delayed. Thus, given these dynamics over the course of a trial, interference effects are reduced by selective response inhibition more in slow than in fast responses. Consequently, Simon delta plots should reveal a pattern of reduced interference in slower segments of the RT distribution as the suppression mechanism becomes more fully engaged (de Jong et al., 1994; Ridderinkhof, 2002a,b).

Given that effective selective inhibition results in a more pronounced reduction of interference effects for slow responses, as argued above, then the attenuation of interference effects (i.e., the leveling-off of the delta-plot slope) should be more pronounced in experimental conditions that require selective suppression (Burle et al., 2002; Ridderinkhof, 2002b; Wijnen and Ridderinkhof, 2007; Wylie et al., 2009b). Moreover, the slope value that quantifies the reduction of interference at the slowest segment of the RT distribution serves as a sensitive metric of the proficiency of selective response inhibition (Burle et al., 2002; Ridderinkhof, 2002b; Ridderinkhof et al., 2004c, 2005). On the basis of the assumptions of the temporal dynamics of online control, derived from the activation-suppression model, we take the slope value of the slowest delta-plot segment to best capture the proficiency of selective suppression. This does not mean that the effects of selective suppression may not become evident in earlier RT segments, but this depends on several factors like the strength of initial response capture, the number of RT segments and the strength of the suppression mechanism.

First, we focus on studies that establish the basic validity of interpreting these delta patterns as reflecting selective suppression, and then cover studies that have used delta-plot measures as a probing tool. Several behavioral studies provided proof of principle for the notion that the delta-slope value at the slowest portions of the delta plot varies as a function of experimental manipulations of inhibitory demands. Increasing the relative probability of incompatible trials generally reduces the overall mean Simon effect (Stürmer et al., 2002) and yields stronger selective suppression of locationdriven activation for slower responses, reflected by a steeper negative-going delta-plot slope as RT slows (Ridderinkhof, 2002b). Similarly, a dramatic negative-going delta slope occurs much earlier in the RT distribution on trials that follow incompatible trials compared to trials following compatible trials (Ridderinkhof, 2002b; Wylie et al., 2010). A compelling illustration that confirms the link between delta-slope values and inhibitory control is provided by a study comparing Flanker effects in children diagnosed with AD/HD and matched controls (Ridderinkhof et al., 2005). Although overall group differences in mean flanker effects were marginal, delta-plot analyses uncovered a pattern of less proficient interference control among children with AD/HD. Moreover, among children with $\mathrm{AD} / \mathrm{HD}$, delta slopes were more negative-going after administration of methylphenidate, suggesting an ameliorative effect on the response inhibition deficit. These findings support current theories that emphasize a deficit in response inhibition as a fundamental neurocognitive impairment in AD/HD (Nigg, 2001).

The studies above confirmed the notion that variations in the temporal dynamics of interference effects reflect demands on selective suppression that are sensitive to experimental manipulations as well as group differences in inhibitory proficiency. The activation-suppression model inspired several experiments that used the delta-plot technique to further probe variations in selective suppression. For example, Burle et al. (2005) explored the time-course of selective suppression by systematically varying the temporal overlap between the onsets of the relevant and irrelevant stimulus dimensions. They revealed a clear reversal of the interference effect (i.e., negative slope values) if the irrelevant information was presented before the relevant information; long RTs were associated with a negative interference effect, consistent with more effective suppression. Furthermore, manipulation of the spatial configuration of stimuli in a Simon task (e.g., vertical versus 
horizontal displacement) yields a negative-going delta-plot pattern for horizontally displaced stimuli, but a positive slope for vertically displaced stimuli (Wiegand and Wascher, 2005, 2007). This suggested a stronger need for selective suppression when stimuli are presented in the left or right visual field that is directly corresponding to the location of the required response hand. Furthermore, compared to a rest condition, physical exercise reduced the proficiency of selective suppression (Davranche and McMorris, 2009). Finally, selective suppression, as revealed by negative-going delta slopes, is not restricted to manual actions since a similar pattern has been obtained for foot responses (Davranche et al., 2009) and for eye movements (Wijnen and Ridderinkhof, 2007).

Delta-plot analyses are not only sensitive to experimental factors, but also provide a sensitive quantitative metric to study individual differences in the proficiency to suppress interference that arises from the activation of conflicting responses. In their developmental study of participants ranging in age between 19 and 82 years, Juncos-Rabadán et al. (2008) applied the delta-plot technique on data obtained with a variant of the Simon task. They observed that for 19- to 26-year-old participants the magnitude of the Simon interference effect decreased with response speed (i.e., showing a negative-going delta slope). However, interference increased as a function of RT among 70- to 82-year-olds. This developmental pattern supports the conclusion that selective suppression declines with older age. Focusing on children, Bub et al. (2006) presented the standard Stroop task (Stroop, 1935) to a group of children between 7 and 11 years of age. Interference was induced by having the children name the print color of a (non-color) word while resisting the prepotent tendency to read the word aloud. Younger children displayed increased mean interference on conflict trials, both in terms of increased error rates and RT slowing. However, plotting Stroop interference effects as a function of response speed revealed a reduction of the interference effect for slower responses that was at least as pronounced for younger as for older children (Bub et al., 2006). Taken together, these developmental studies indicate that the proficiency of selective suppression emerges across the early years of development but is vulnerable to decline with advanced age. In addition, several clinical studies that employed the delta-plot technique have pointed to increased difficulty in resolving interference in several populations, such as patients diagnosed with mild cognitive impairment (Wylie et al., 2007) and Parkinson's disease (see Figures 5A,B; Wylie et al., 2009a,b, 2010).

\section{ALTERNATIVE ACCOUNTS}

The behavioral studies discussed above illustrate the effectiveness of distributional analyses of response errors and response speed to quantify the temporal aspects and to dissociate the activation of incorrect responses and its subsequent selective suppression. The activation-suppression model provides a powerful framework to interpret the present results; the patterns derived from distributional analyses conform accurately to the model's predictions and the results point to the hypothesized variations in inhibitory control as a function of experimental factors. However, we recognize that accounts other than the activation-suppression model may potentially explain the reduction of interference effects over time. For example, the leveling-off of interference effects toward the slow end of the RT distribution might result from a process of passive decay of the initial response activation by the direct route, instead of the activation of a top-down suppression mechanism (Hommel, 1994). Although passive decay might potentially result in an attenuation of interference effects, mere decay is unlikely to account for reversals of the Simon effect. Such negative Simon interference effects are evident in the delta plot for a group of healthy control participants (see Figure 5A), and for both controls and Parkinson patients when plotting the Simon delta values for trials that are preceded by incompatible trials (e.g., Wylie et al., 2010). An interference effect below 0 would require an active process of suppression of the incorrect response activation during incompatible trials that leads to faster response selection on these trials. Similarly, computational modeling architectures that do not take into account active suppression units have not been very successful in describing negative interference effects (e.g., Davelaar, 2008).

Support for the involvement of active top-down suppression to resolve conflict is provided by psychophysiological experiments that focused on neurocognitive mechanisms that underlie the expression and suppression of impulsive actions. The next sections provide a selective review of studies that used individual differences in RT-distribution parameters to probe the efficiency and temporal dynamics of selective response inhibition and to guide the detailed analysis of neuroimaging data.

\section{EMG ERRORS REPRESENT DETECTED AND CORRECTED IMPULSIVE ACTIVATION}

The notion of incorrect response activation refers to a covert process that is not directly observable in behavior. Electrophysiological measures provide a useful tool to reveal such covert processes. In this respect, recording the electromyographic activity of the muscles involved in response execution has proved useful (Coles et al., 1985; Smid et al., 1990; Burle et al., 2002). Compared to other potential measures of response activation like electroencephalography (EEG), EMG provides several major advantages. First, EMG activity can unequivocally be associated with motor responses, whereas it is harder to map EEG measures directly to motor responses because of volume conduction. Second, thanks to its excellent signal-tonoise ratio, EMG activity can be detected on a trial-by-trial basis, hence avoiding spurious effects of averaging (see Burle et al., 2008; Figure 6 for an example).

\section{ACTIVATION OF INCORRECT ACTIONS: PARTIAL EMG ERRORS}

In the context of conflict tasks, EMG recordings have revealed that on a proportion of trials in which the correct response was issued, phasic EMG activities occurred in both the correct and incorrect response agonist muscles (Coles et al., 1985; Eriksen et al., 1985). Following these seminal papers, incorrect EMG was studied in more detail by Smid et al. (1990) who distinguished trials on which the incorrect EMG occurred before the correct response, from trials on which the incorrect EMG occurred after it (see Figure 6A). They reported that only the first trial category was contributing to conflict effects, making this category of special interest, whereas the second category likely reflects mere motor noise. Follow-up studies, although sparse, have largely confirmed the particular interest of such "partial error" trials. Table 1 presents the percentage of partial errors on compatible and incompatible trials observed across different tasks and studies. Although the absolute percentage 


\section{A Partial EMG Error}

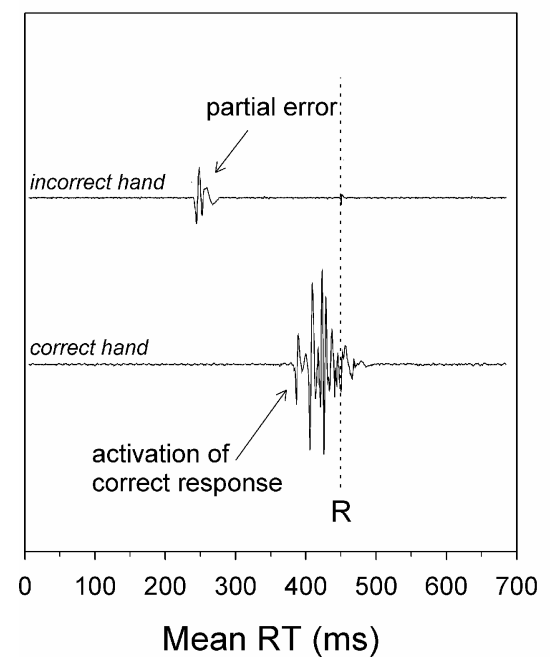

FIGURE 6 | Partial EMG errors. (A) Electromyogram (EMG) from muscles controlling the incorrect (upper trace) and the correct response (lower trace). The vertical dashed line indicates the mechanical button-press response. The correct overt response was preceded by partial EMG activity in the muscle that controls
B Partial EMG Errors $x$ Time

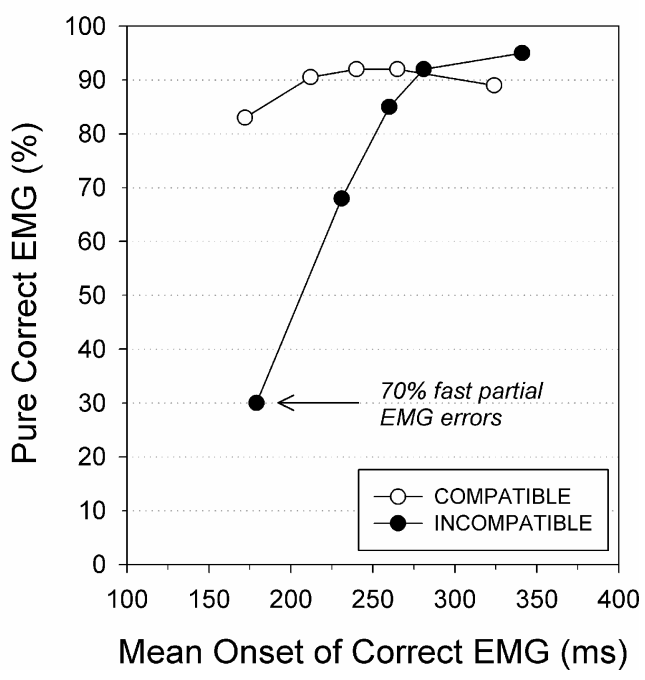

the incorrect response that is too weak to trigger an overt error. (B) Fast responses on incompatible trials yield a high percentage of stimulus-driven partial EMG errors of the incorrect hand before the correct button press is emitted. Re-analyzed data published by Burle et al. (2002).

Table 1 | Percentage of partial EMG errors for compatible and incompatible trials reported in the literature.

\section{Compatible}

(\%)

$(\%)$

\begin{tabular}{lrr}
\hline SIMON TASK & & \\
Burle and Bonnet (1999) & 15 & 17 \\
Hasbroucq et al. (1999) & 10 & 7 \\
Burle et al. (2002, exp. 1) & 4 & 13 \\
Burle et al. (2002, exp. 2) & 5 & 26 \\
Hasbroucq et al. (2009, exp. 1) & 15 & 23 \\
Hasbroucq et al. (2009, exp. 2) & 17 & 23 \\
ERIKSEN TASK & 8 & 22 \\
Smid et al. (1990) & 14 & 27 \\
Burle et al. (2008) & 12 & 26 \\
Burle and Hasbroucq (submitted, exp. 1) & 20 \\
Burle and Hasbroucq (submitted, exp. 2) & 20 \\
FITTSTASK & 11 & 19 \\
Hasbroucq et al. (2001) & & \\
\hline
\end{tabular}

A few other studies recorded partial errors in conflict tasks but did not report percentages.

may vary from study to study, it is clear that the percentage of partial EMG errors is always larger on incompatible compared to compatible trials. Such an increase in incorrect response activation on incompatible trials directly supports a key assumption of the dual-route model (Kornblum et al., 1990), namely that the irrelevant aspect (position, flankers, etc.), more or less automatically, activates the response to which it is associated, either by instruction (e.g., in the Eriksen task) or by habit strength (e.g., in the Simon and Fitts tasks).
Further arguments supporting the idea that those incorrect response activations are true partial errors come from EEG and sequential effect analysis. First, incorrect EMG occurring before the response (i.e., partial errors) are followed by a small but robust error negativity (Scheffers et al., 1996; Vidal et al., 2000; Masaki et al., 2007; Burle et al., 2008; Roger et al., 2010), whereas incorrect EMG occurring after the correct response are not (Allain et al., 2004). Furthermore, we recently showed that, like overt errors, partial errors are followed by response slowing, expressed as a RT lengthening after a trial containing a partial error (although this post-error slowing is much smaller than after an overt error; Allain et al., 2009).

Besides this rather "static" view of incorrect response activation, a more dynamic view is obtained by the CAF that plots the percentage of correct responses as a function of the latency of the response. Instead of taking into account only overt behavior (i.e., $\mathrm{RT}$ ), one can plot the frequency of incorrect response activation (EMG measure), as a function of the activation latency. An example of such an analysis in a Simon task is presented in Figure 6B. As for "traditional" CAFs, it appears that relatively slow response activations are mainly correct, with an asymptote around 5\% errors, but that fast response activations are much less accurate. Interestingly, CAFs based on partial-errors reveal that fast incorrect response activations are clearly above chance level on incompatible trials (70\% partial EMG errors), whereas classical CAFs of RT often indicate chance level accuracy. Such a high percentage of incorrect response activation discards the possibility that partial errors simply reflect guesses, since guesses should yield accuracy rates around chance level. To the contrary, such a pattern clearly indicates that these incorrect EMGs are mainly stimulus driven, and hence reflect the activation of response by the irrelevant dimension of the stimulation display. 


\section{SELECTIVE SUPPRESSION OF INCORRECTLY ACTIVATED EMG RESPONSES}

Since partial EMG errors truly reflect stimulus-driven incorrect response activation, as argued above, they offer an opportunity to directly test the response suppression hypothesis. Indeed, on trials with a partial EMG error, the incorrect response has been largely activated (as it reached late stages of the reaction process) but was nonetheless appropriately suppressed, since the correct response was finally issued. According to the activation-suppression model (Ridderinkhof, 2002a), one should thus predict steeper negativegoing delta-plots for trials containing partial EMG errors. This prediction was tested directly (Burle et al., 2002), and the results are presented in Figure 7. As one can see, when all trials are taken into account, that is pooled across trials with and trials without partial EMG, the delta-plot slopes are negative-going (black circles) as usually reported in the literature. Interestingly, when excluding trials with partial EMG activation of the incorrect response, the last delta-plot slope is much less negative going (white circles). This finding supports the idea that the reduction of the interference effect, reflected by the delta slope, is related to the detection and suppression of incorrect responses that are activated up to the level of the incorrect muscles.

In sum, analysis of EMG patterns has provided direct evidence for the subthreshold activation of impulsive actions up to the level of the muscles that control the motor response and its subsequent selective suppression. The following section presents an overview of functional as well as structural imaging studies that used the parameters generated by the activation-suppression model in search of brain areas that are associated with activation and selective suppression.

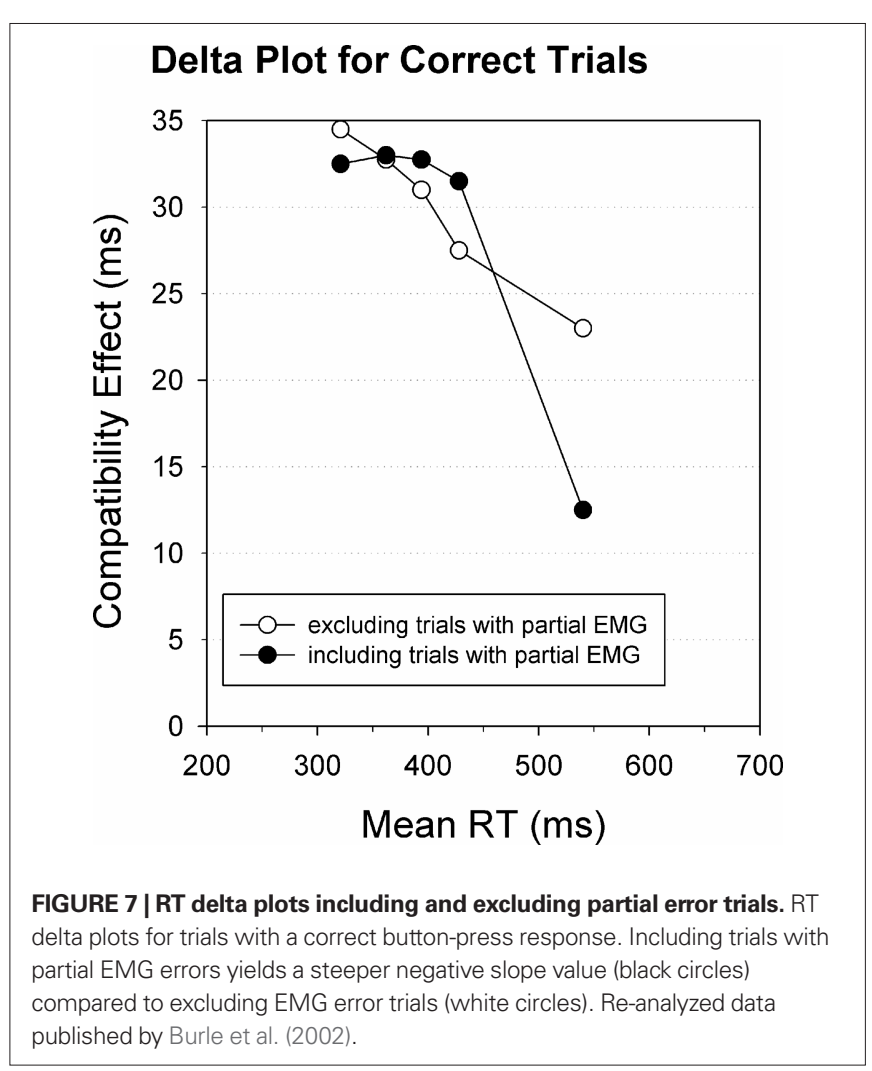

\section{NEURAL MECHANISMS UNDERLYING ACTIVATION AND INTERFERENCE CONTROL}

Despite the wealth of literature on conflict tasks such as the Stroop task and Eriksen flanker variants, neuroimaging studies using the Simon task are still relatively rare. The activation pattern most commonly reported in conflict tasks consists of a fronto-parietal and fronto-striatal network, including the medial frontal cortex, the lateral prefrontal cortex, the posterior parietal cortex, as well as the dorsal striatum and the subthalamic nucleus (STN) (e.g., Pardo et al., 1990; Peterson et al., 2002; Hazeltine et al., 2003; Schumacher et al., 2007; for a meta-analysis see Nee et al., 2007; for a reviews see Ridderinkhof et al., 2004b, 2010). More specifically, processes like monitoring of response conflict and response selection are believed to play a crucial role in task performance and have been associated with functioning of the medial frontal cortex (Ridderinkhof et al., 2004a). Others have pointed to the essential role of the lateral prefrontal cortex in implementing top-down control after experiencing response conflict or suppressing unwanted response tendencies (Kerns et al., 2004). Finally, the posterior parietal cortex subserves visuo-motor transformations via middle occipital and inferior temporal cortices important for attentional control (Kastner and Ungerleider, 2000).

A central question is to what extent this general network reveals task-specific or task-independent selection, control, and suppression of actions (see also Schumacher et al., 2003). The Stroop task engages several putative control processes, including monitoring of response conflict, selection between competing responses, and selective response inhibition, but the relevant contrast in this task may capture other processes as well (e.g., conflict at the semantic level). A recent quantitative meta-analysis by Nee et al. (2007) that included fMRI data from several Stroop tasks revealed a strong left lateralized network including the left middle frontal gyrus, the insula, as well as the left posterior parietal cortex. Contrary to Stroop stimuli, the target and flanker stimuli in the Eriksen flanker paradigm are presented within the same stimulus dimension without requiring semantic processes. With respect to Flanker task performance, meta-analyses shows mainly right lateralized activation clusters in the middle frontal gyrus and the insula (Nee et al., 2007). Interestingly, these patterns are somewhat comparable to activations found with stimulus-response compatibility tasks such as the Simon task (Peterson et al., 2002; Liu et al., 2004).

\section{DATA-DRIVEN VERSUS COVARIATE-BASED FMRI APPROACH}

The heterogeneity of the activation patterns on conflict tasks reported above might be driven by two underlying sources of variance. First, most studies do not take into account considerable differences in interference control across individuals. When activation levels are pooled over participants, the variance related to individual differences may mask the detection of activation patterns that emerge when contrasting incompatible and compatible trial types. The second source of variance that might have contributed to inconsistent findings across fMRI reports relates to the calculation contrasting brain activation derived from incompatible trials and compatible trials, i.e., the mean interference effect on brain activity. As outlined in the previous sections, overall mean interference effects mask the vital dynamic aspects of response impulse activation followed by selective suppression of this activation. A 
potentially more effective approach is to relate brain activation with specific behavioral parameters as specified by the activation-suppression model and as revealed by distributional analyses. Recent neuroimaging studies by Forstmann et al. (2008a,b) adopted this approach by focusing on individual differences in interference control, taking into account the temporal dynamics of activation and subsequent suppression of incorrect response activation. Twenty-four participants performed a variant of the Simon task (see Figure 1) in which the designated response is indicated by an aspect of the imperative signal (its color), but competing response impulses were elicited by the task-irrelevant spatial location of the stimulus. In a separate neutral block without spatial interference, the participants responded to the color of a circle presented in the middle of the screen. The following sections provide answers to questions like, "does the activation of certain brain areas covary with the strength of initial capture by an incorrect response impulse?" and "are there dissociable brain areas whose activation is linked to the proficiency of selective suppression as revealed by negative-going delta slopes?"

\section{IMPULSIVE ERRORS: ACTIVATION IN THE RIGHT PRE-SMA}

In line with the predictions of the activation-suppression model, fast responses on conflict trials were considerably more prone to errors than slower incompatible responses. The error slope value derived from the fast RT segment for incompatible trials was used to infer the individual's susceptibility to making fast, impulsive errors (red slope depicted in Figure 8, upper panel). Individuals showing a relatively steeper positive-going error slope value for the fast RT segment had a stronger tendency to emit fast and unintentional impulsive responses that are driven by the irrelevant stimulus dimension. Analyses of brain activation focused on the contrast between incompatible versus neutral trials. To study the neural substrates of impulsive action selection, individual slope values derived from the fast segment of the CAF for incompatible responses were entered as covariates in the fMRI regression analyses. Steeper positive-going error slopes were associated with enhanced activation in the right pre-supplementary motor area (pre-SMA, see Figure 8, middle panel). The pre-SMA was found to be active on correct incompatible trials that are associated with increased error rates (Forstmann et al., 2008b). This pattern indicates that individuals who are highly susceptible to making fast impulsive errors show increased pre-SMA activity reflecting the elevated need to select a correct response in conflicting situations. Notably, the relation between errors and percent signal change derived from the preSMA was evident only for the fast RT segment, and was absent for slower responses, a dynamic pattern that is in line with the tenets of the activation-suppression model.

\section{SELECTIVE SUPPRESSION: ACTIVATION IN THE RIGHT IFC}

Next, the covariate-based fMRI approach was used to identify the neurocognitive correlates of selective response inhibition to resolve conflict arising from the activation of involuntary action impulses. To this end, the individual reduction of the Simon effect was quantified as a function of RT; the value of the delta slope derived from the slow segment of the RT distribution (red slope depicted in Figure 8, upper-right panel). Entering individual delta-slope values into the fMRI regression analyses revealed significant activations in the right inferior frontal cortex (IFC, BA 44) bordering the right anterior insula (Forstmann et al., 2008b). This pattern reveals that individuals showing relatively negative-going delta-slope values derived from the slowest segment of the RT distribution present enhanced activation in the right IFC. The specificity of taking into account the temporal aspects of interference control is underscored by the null-results of two additional analyses. First, the two delta-slope values derived from faster RT segments did not co-vary significantly with brain activation. A second null finding was obtained when individual mean interference effects (i.e., overall average Simon effects) were entered as covariates in the fMRI regression model. These findings provide direct evidence that activation in the right IFC varies as a function of individual efficacy to resolve response conflict that arises from involuntary action tendencies.

\section{STRUCTURAL CONTRIBUTIONS TO SELECTIVE SUPPRESSION}

The fMRI studies discussed above capitalized on individual differences in the activation and selective suppression of incorrect responses and identified the involvement of the pre-SMA and the right IFC, respectively. This section highlights a study that has specified the relationship between behavioral parameters derived from the RT distributions and the structural integrity of the brain. The specific fMRI activation patterns in the pre-SMA and right IFC were replicated and extended in a second covariate-based study using a modified version of the Simon task (Forstmann et al., 2008a). Again, individual distribution parameters (i.e., the delta-slope value of the slow RT segment) predicted activation in the right IFC, confirming the close match between the proficiency of selective suppression and right IFC function. As a novel approach, structural data on the density of coherent white matter tracts were obtained using diffusion tensor imaging (DTI). The dependent measure of interest was fractional anisotropy (FA), reflecting the degree of diffusion anisotropy within a voxel that depends on microstructural features of the tissue that includes fiber properties and tract coherence. First, individual RT distribution parameters were used to classify subgroups of good and poor inhibitors based on a median split of the slopes from the slowest segment of the delta plot. The connectivity data revealed higher FA values in the right anterior part of the inferior fronto-occipital fasciculus (FOF) and in the precuneus for good versus poor performers (see Figure 9). This pattern reveals a tight linkage between brain structure (FA values in the anterior part of the FOF) and the proficiency of selective response inhibition. This pattern of connectivity differences in the anterior part of the FOF closely resembled the corresponding pattern for functional activation of the right IFC. Finally, our third hypothesis stated that the right IFC BOLD activation and the right IFC local structural differences should correlate positively. This hypothesis was also confirmed by the present data pattern (Figure 9). These finding reflect a systematic linkage of individual differences in selective response inhibition at the behavioral, functional, and structural level, as supported by independent techniques (Aron et al., 2007; Forstmann et al., 2008a,b; Neubert et al., 2010).

\section{THE SUBTHALAMIC NUCLEUS: DEEP-BRAIN STIMULATION}

According to many contemporary views, the implementation of action selection and inhibition processes is accomplished by cortical-subcortical interactions. To this point, the reviewed studies 

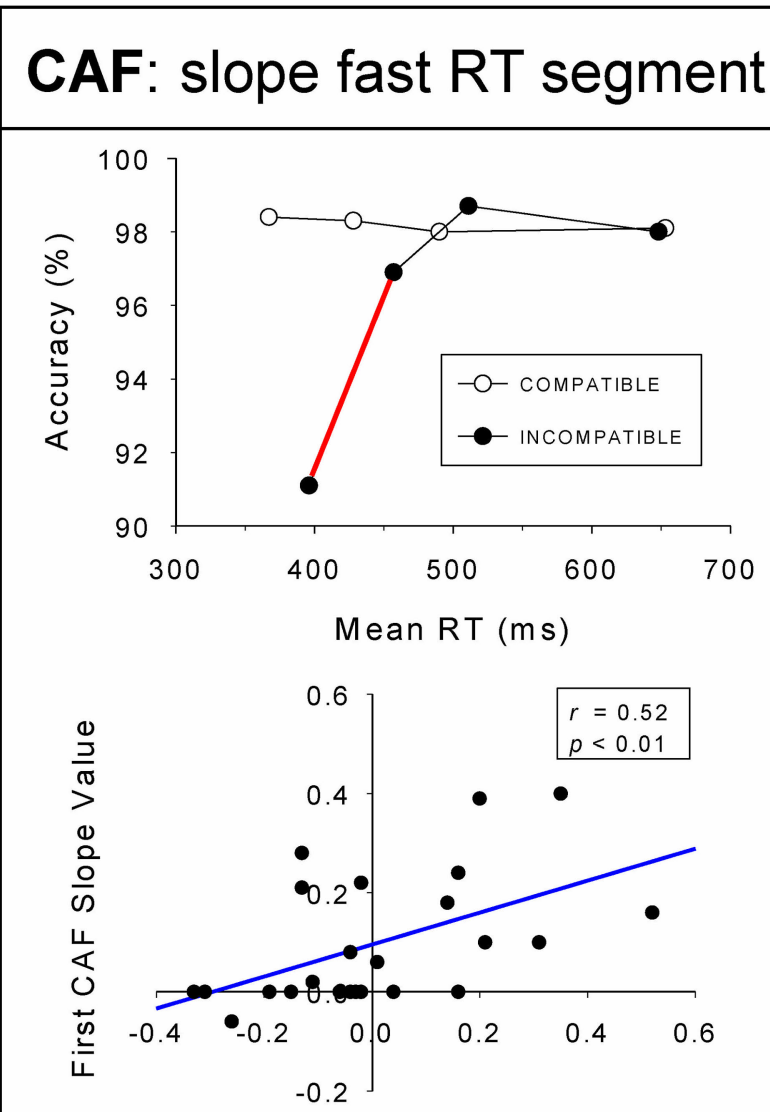

$\%$ signal change pre-SMA

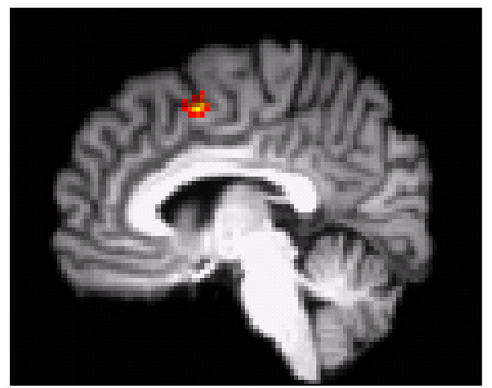

pre-SMA $(x=-4 ; y=6 ; z=52)$

\section{DELTA: slope slow RT segment}
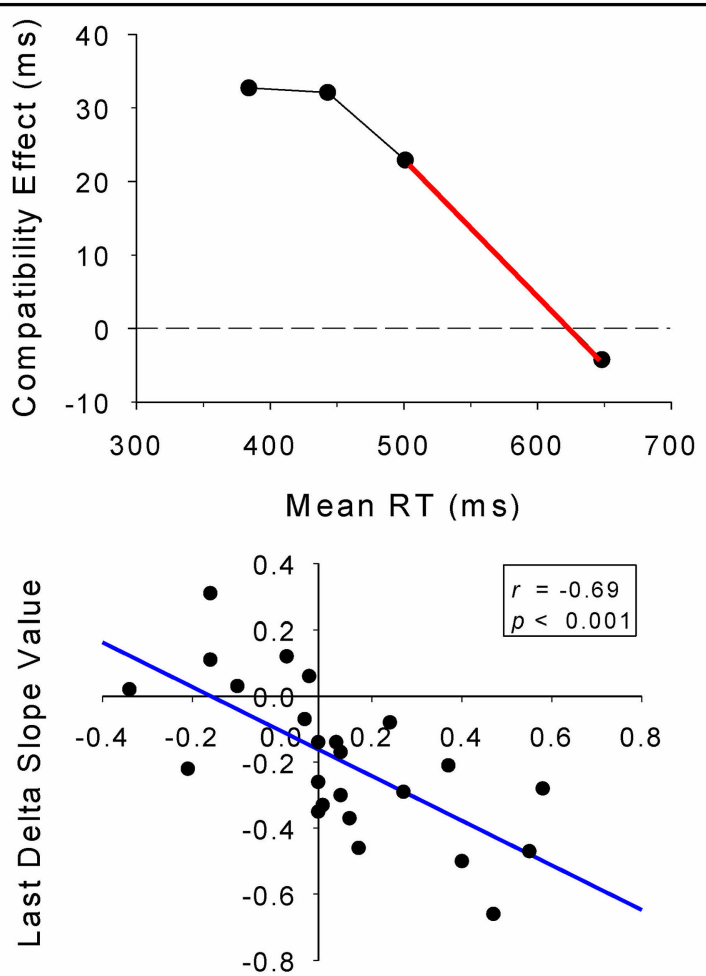

$\%$ signal change right IFC

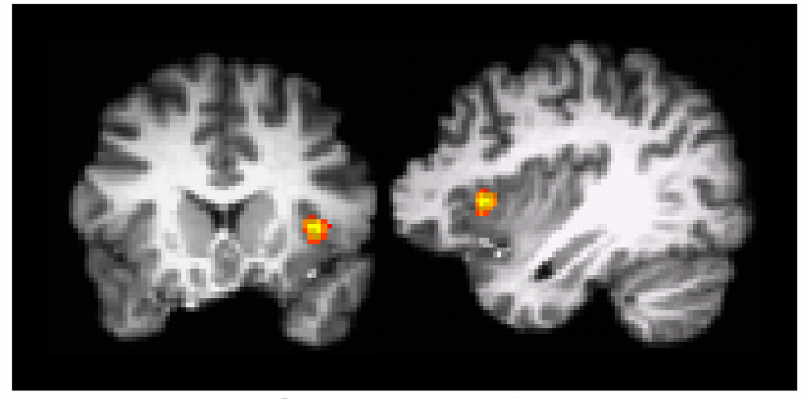

right IFC $(x=38 ; y=20 ; z=4)$
FIGURE 8 | Slope values predict brain activation. Covariate analyses with individual behavioral parameters from 24 participants. Top row displays the average conditional accuracy function (CAF) and average delta slopes separated in fast, middle, and slow RT segments. Middle row left shows Pearson correlations between the first CAF slope for incompatible trials and the $\%$ signal change ( $x$-axis) derived from the pre-SMA. Middle row right shows Pearson correlations between the last delta slope ( $y$-axis) and \% signal change ( $x$-axis) derived from the right IFC. Figure modified after Forstmann et al. (2008b). indicate key involvement of cortical activity in pre-SMA and right IFC in action selection and selective inhibition of action, respectively. Both of these cortical areas project directly to the basal ganglia, a network of subcortical structures whose involvement in releasing or preventing movement has been firmly established (see Mink, 1996, for a review). Of particular interest in the present review are so-called hyper-direct pathways connecting pre-SMA and right IFC to the STN that have been postulated to play an important role in action control processes. As a result, the STN may be unique among basal ganglia structures in its involvement in both rapid action selection and top-down selective suppression processes (Frank, 2006; van den Wildenberg et al., 2006).

Studies of patients diagnosed with Parkinson's disease who have been surgically implanted with deep-brain stimulation (DBS) electrodes in the STN offer a unique opportunity to examine the role of STN modulation on action selection and inhibition processes. In a recent study, we used distributional analyses of the Simon effect to investigate the role of STN stimulation in medicated Parkinson's 


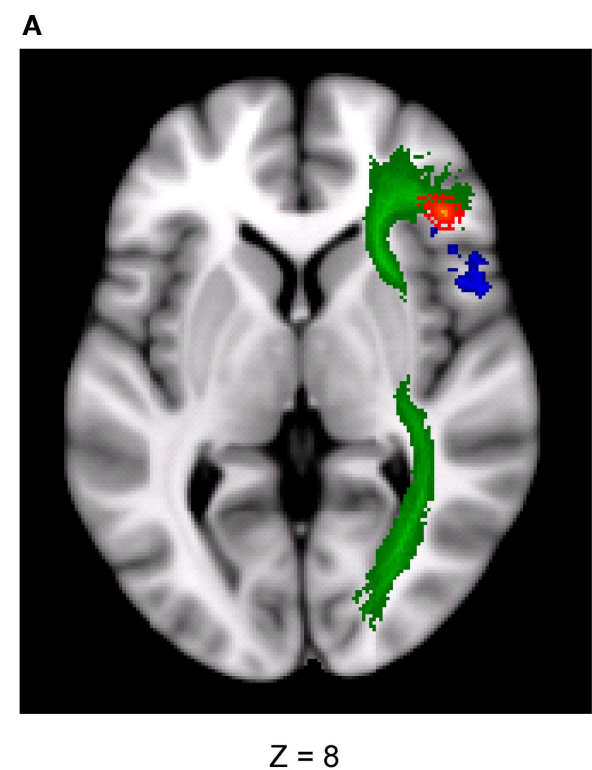

FIGURE 9 | Relation between delta slope, rIFC activation, and structural measures. (A) Axial view showing the inferior fronto-occipital fasciculus (FOF, in green). Contrasting good versus poor inhibitors shows increased connectivity in the anterior FOF (in red) and increased BOLD activation in the right IFC (blue). (B, upper plot) Pearson correlation between connectivity in

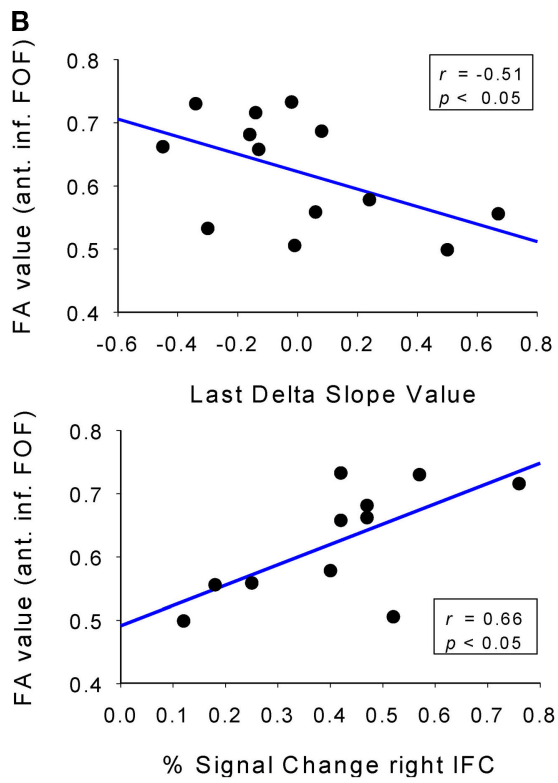

the anterior part of the FOF and slope values derived from the delta plot of the last RT segment. (B, lower plot) Pearson correlation between connectivity in the anterior part of the FOF and percentage BOLD signal change derived from the right IFC. Figure modified after Forstmann et al. (2008a). patients on the strength of capture by response impulses and the proficiency of selective suppression (Wylie et al., 2010). Parkinson's patients performed the Simon task under conditions in which their STN was and was not being stimulated. Whereas mean values did not disclose differences in interference effects attributable to STN stimulation, distributional analyses revealed important effects on response capture and selective suppression dynamics. When the STN was not being stimulated, patients showed similar rates of fast errors as healthy controls, suggesting normal susceptibility to impulsive behavior. However, Parkinson's patients were less proficient at suppressing the interference with goal-directed action caused by the activation of the incorrect response impulse (i.e., they showed less reduction of the Simon effect as RT slowed), a finding that replicated previous results in medicated Parkinson's disease patients who were not treated with STN DBS (Wylie et al., 2010). Turning on stimulation to the STN produced two significant effects on performance. First, patients reacted more impulsively compared to when their STN stimulation was turned off as well as compared to healthy controls. That is, they showed an appreciable increase in fast errors on conflict trials. Second, on conflict trials that were responded to correctly, STN stimulation improved the patients' ability to suppress the interference that was generated by the activation of the impulsive action. Thus, STN stimulation both increased impulsive action selection yet improved inhibitory control over the conflict produced by impulsive action tendencies that did not lead to overt response errors. These seemingly paradoxical effects can be understood within the activation-suppression model by asserting that STN stimulation influences two temporally and neurally dissociable aspects of conflict processing, an initial phase related to an individual's susceptibility to response capture by incorrect response impulses and a later phase associated with the engagement and build-up of top-down, selective inhibitory control over conflicting responses. These results fit well with human and animal studies that have linked STN activity and its manipulation to changes in both impulsive behavior and response suppression across a variety of experimental tasks (Eagle et al., 2008; Ballanger et al., 2009). It will be important for future imaging studies to investigate the potential role of pre-SMA and right IFC projections to STN as mediators of action selection and inhibition circuits, respectively.

\section{CONCLUSION}

This selective review of behavioral and psychophysiological correlates of performance on conflict tasks shows that distributional analyses can isolate vital processes that are involved in the cognitive control of interference effects with greater precision than can mean performance measures. A merit of the activation-suppression model is the functional dissociation between the susceptibility of making fast impulsive actions (response capture), and subsequent cognitive control that is engaged to keep unwanted action activation in check to facilitate the selection of the correct action (selective action inhibition). The first process is indexed by a pattern of fast errors on incompatible trials (depicted in a CAF). The second process is reflected by a decrease in the interference effect for slower responses (depicted in a delta plot). Importantly, the activation-suppression model yields behavioral parameters that are sensitive to these temporal dynamics and, as such, provided a useful tool to study the impact of experimental factors and individual differences in action control during conflict.

Model-based analyses that capitalize on individual differences in response capture and subsequent suppression revealed structural as well as functional differences related to specific brain 
regions. Parameters indicating response capture and selective inhibition in the Simon task have guided neurocognitive studies highlighting the pre-SMA and right IFC as key nodes for action selection. Direct connections between the pre-SMA and basal ganglia structures (most prominently the anterior dorsal striatum and the STN) serve to keep basal ganglia output in check until intention-driven action selection has completed. Extraneous, impulsive action affordances may capture the action system non-deliberately. When multiple stimulus-action association alternatives compete for activation, the demands on action control are highest, and selecting the appropriate action engages stronger activation of the pre-SMA compared to when response capture is absent. The pre-SMA may act as an actionselection director, modulating the action-selection gate through which the available action affordances are translated into actual actions (Mars et al., 2009; Ridderinkhof et al., 2010). The right IFC is recruited to signal the need for implementation of selective response inhibition (Forstmann et al., 2008a,b, see also Aron et al., 2007; Verbruggen et al., 2010). In particular, individual differences in the efficiency to implement inhibitory control in humans are associated consistently with functional and structural

\section{REFERENCES}

Allain, S., Burle, B., Hasbroucq, T., and Vidal, F. (2009). Sequential adjustments before and after partial errors. Psychon. Bull. Rev. 16, 356-362.

Allain, S., Carbonnell, L., Burle, B., Hasbroucq, T., and Vidal, F. (2004). "Electromyographic activities and the Ne," in Errors, Conflicts, and the Brain: Current Opinions on Performance Monitoring, eds M. Ullsperger and M. Falkenstein (Leipzig: MPI of Cognitive Neuroscience), 21-27.

Aron, A. R., Behrens, T. E., Smith, S., Frank, M. J., and Poldrack, R. A. (2007). Triangulating a cognitive control network using diffusion-weighted magnetic resonance imaging (MRI) and functional MRI. J. Neurosci. 27, 3743-3752.

Ballanger, B., van Eimeren, T., Moro, E., Lozano,A.M., Hamani, C., Boulinguez, P., Pellecchia, G., Houle. S., Poon, Y. Y., Lang, A. E., and Strafella, A. P. (2009). Stimulation of the subthalamic nucleus and impulsivity: release your horses. Ann. Neurol. 66, 817-824.

Band, G. P. H., Ridderinkhof, K. R., and van der Molen, M. W. (2003). Speed-accuracy modulation in case of conflict: the roles of activation and inhibition. Psychol. Res. 67, 266-279.

Bub, D. N., Masson, M.E. J., and Lalonde, C. E. (2006). Cognitive control in children: Stroop interference and suppression of word reading. Psychol. Sci. 17, 351-357.

Burle, B., and Bonnet, M. (1999). What's an internal clock for? From temporal information processing to temporal processing of information. Behav. Processes 45, 59-72.
Burle, B., Possamaï, C. A., Vidal, F., Bonnet, M., and Hasbroucq, T. (2002). Executive control in the Simon effect: an electromyographic and distributional analysis. Psychol. Res. 66, 324-336.

Burle, B., Roger, C., Allain, S., Vidal, F., and Hasbroucq, T. (2008). Error negativity does not reflect conflict: a re-appraisal of conflict monitoring and anterior cingulate cortex activity. J. Cogn. Neurosci. 20, 1637-1655.

Burle, B., van den Wildenberg, W. P. M., and Ridderinkhof, K. R. (2005). Dynamics of facilitation and interference in cue-priming and Simon tasks. Eur. J. Cogn. Psychol. 17, 619-641.

Coles, M.G.H., Gratton, G., Bashore, T.R., Eriksen, C.W., and Donchin, E. (1985). A psychophysiological investigation of the continuous flow of human information processing. J. Exp. Psychol. Hum. Percept. Perform. 11, 529-552.

Davelaar, E. J. (2008). A computational study of conflict-monitoring at two levels of processing: reaction time distributional analyses and hemodynamic responses. Brain Res. 1202, 109-119.

Davranche, K., and McMorris T. (2009). Specific effects of acute moderate exercise on cognitive control. Brain Cogn. 69, 565-570.

Davranche, K., Paleresompoulle, D., Pernaud, R., Labarelle, J., and Hasbroucq, T. (2009). Decision making in elite white-water athletes paddling on a kayak ergometer. J. Sport Exerc. Psychol. 31, 554-565.

de Jong, R., Liang, C. C., and Lauber, E. (1994). Conditional and unconditional automaticity: a dual-process model of effects of spatial stimulus-response

differences in the right IFC. In turn, the basal ganglia serve to keep all responses in check until the final green signal is received from upstream (Frank, 2006; van den Wildenberg et al., 2006; Wylie et al., 2010). In later processing stages, suppression of unwanted responses may be evident at the level of the primary motor cortex (van den Wildenberg et al., 2010) and even shows in suppression of subthreshold EMG activity in incorrectly activated response muscles (Burle et al., 2002).

\section{ACKNOWLEDGMENTS}

Wery P. M. van den Wildenberg, Birte U. Forstmann, and K. Richard Ridderinkhof were supported by grants from the Netherlands Organization for Scientific Research (NWO). This work was supported by a grant (K23AG028750) awarded to Scott A. Wylie by the National Institute on Aging (the content is solely the responsibility of the authors and does not necessarily represent the official views of the National Institute On Aging or the National Institutes of Health). Borís Burle was supported by a grant from the European Research Council under the European Community's Seventh Framework Program (FP7/2007-2013 Grant Agreement no. 241077).

correspondence. J. Exp. Psychol. Hum. Percept. Perform. 20, 731-750.

Eagle, D. M., Baunez, C., Hutcheson, D. M., Lehmann, O., Shah, A. P., and Robbins, T. W. (2008). Stop-signal reaction-time task performance: role of prefrontal cortex and subthalamic nucleus. Cereb. Cortex 18, 178-188.

Eimer, M., Hommel, B., and Prinz, W. (1995).S-R compatibility and response selection. Acta Psychol. 90, 301-313.

Eriksen, B. A., and Eriksen, C. W. (1974) Effects of noise letters upon the identification of target letters in a nonsearch task. Percept. Psychophys. 16, 143-149.

Eriksen, C. W., Coles, M. G. H., Morris, L. R., and O'Hara, W. P. (1985). An electromyographic examination of response competition. Bull. Psychon. Soc. 23, 165-168.

Forstmann, B. U., Jahfari, S., Scholte, H. S., Wolfensteller, U., van den Wildenberg, W. P. M., and Ridderinkhof, K. R. (2008a). Function and structure of the right inferior frontal cortex predict individual differences in response inhibition: a model-based approach.J. Neurosci. 28, 9790-9796.

Forstmann, B. U., van den Wildenberg, W. P.M., and Ridderinkhof, K. R. (2008b). Neural mechanisms, temporal dynamics, and individual differences in interference control. J. Cogn. Neurosci. 20, 1854-1865.

Frank, M. J. (2006). Hold your horses: a dynamic computational role for the subthalamic nucleus in decision making. Neural Netw. 19, 1120-1136.

Hasbroucq, T., Burle, B., Akamatsu, M., Vidal, and F., and Possamaï, C. A.
(2001). An electromyographic investigation of the effect of stimulusresponse mapping on choice reaction time. Psychophysiology 38, 157-162.

Hasbroucq, T., Burle, B., Vidal, F., and Possamaï, C.A. (2009). Stimulus-hand correspondence and direct response activation: an electromyographic analysis. Psychophysiology 46, 1160-1169.

Hasbroucq, T., Possamaï, C. A., Bonnet, M., and Vidal, F. (1999). Effect of the irrelevant location of the response signal on choice reaction time: an electromyographic study in humans. Psychophysiology 36, 522-526.

Hazeltine, E., Bunge, S. A., Scanlon, M. D., and Gabrieli, J. D. E. (2003). Materialdependent and material-independent selection processes in the frontal and parietal lobes: an event-related fMRI investigation of response competition. Neuropsychologia 41, 1208-1217.

Hommel, B. (1994). Spontaneous decay of response code activation. Psychol. Res. 56, 261-268.

Juncos-Rabadán, O. J., Pereiro, A. X., and Facal, D. (2008). Cognitive interference and aging: insight from a spatial stimulus-response consistency task. Acta Psychol. 127, 237-246.

Kastner, S., and Ungerleider, L. G. (2000). Mechanisms of visual attention in the human cortex. Annu. Rev. Neurosci. 23, 315-341.

Kerns, J. G., Cohen, J. D., MacDonald, A. W. III, Cho, R. Y., Stenger, V. A., and Carter, C.S. (2004). Anterior cingulate conflict monitoring and adjustments in control. Science 303, 1023-1026.

Kornblum, S. (1994). The way irrelevant dimensions are processed depends 
on what they overlap with: the case of Stroop- and Simon-like stimuli. Psychol. Res. 56, 130-135.

Kornblum, S., Hasbroucq, T., and Osman, A. (1990). Dimensional overlap: cognitive basis for stimulus-response compatibility: a model and taxonomy. Psychol. Rev. 97, 253-270.

Liu, X., Banich, M. T., Jacobson, B. L., and Tanabe, J. L. (2004). Common and distinct neural substrates of attentional control in an integrated Simon and spatial Stroop task as assessed by event-related fMRI. Neuroimage 22, 1097-1106.

Luce, R. D. (1986). Response Times: Their Role in Inferring Elementary Mental Organization. New York: Oxford Science Publications.

Mars, R. B., Klein, M. C., Neubert, F. X., Olivier, E., Buch, E. R., Boorman, E. D., and Rushworth, M. F. (2009). Shortlatency influence of medial frontal cortex on primary motor cortex during action selection under conflict. $J$. Neurosci. 29, 6926-6931.

Masaki, H., Falkenstein, M., Stürmer, B., Pinkpank, T., and Sommer, W. (2007). Does the error negativity reflect response conflict strength? evidence from a Simon task. Psychophysiology 44, 579-585.

Mink, J. (1996). The basal ganglia: focused selection and inhibition of competing motor programs. Prog. Neurobiol. 50, 381-425.

Nee, D. E., Wager, T. D., and Jonides, J. (2007). Interference resolution: insights from a meta-analysis of neuroimaging tasks. Cogn. Affect. Behav. Neurosci. 7, 1-17.

Neubert, F., Mars, R. B., Buch, E. R., Olivier, E., and Rushworth, M. F. S. (2010). Cortical and subcortical interactions during action reprogramming and their related white matter pathways. Proc. Natl. Acad. Sci. U.S.A. 107, 13240-13245.

Nigg, J. R. (2001). Is AD/HD a disinhibitory disorder? Psychol. Bull. 127, 571-598

Pardo, J. V., Pardo, P. J., Janer, K. W., and Raichle, M. E. (1990). The anterior cingulate cortex mediates processing selection in the Stroop attentional conflict paradigm. Proc. Natl. Acad. Sci. U.S.A. 87, 256-259.

Peterson, B. S., Kane, M. J., Alexander, G. M., Lacadie, C., Skudlarski, P., Leung, H. C., May, J., and Gore, J. C. (2002). An event-related functional MRI study comparing interference effects in the Simon and Stroop tasks. Cogn. Brain Res. 13, 427-240.

Ridderinkhof, K. R. (2002a). "Activation and suppression in conflict tasks: empirical clarification through distributional analyses," in Attention and Performance, Vol. XIX, Common Mechanisms in Perception and Action, eds W. Prinz and B. Hommel (Oxford: Oxford University Press), 494-519.

Ridderinkhof, K. R. (2002b). Micro- and macro-adjustments of task set: activation and suppression in conflict tasks. Psychol. Res. 66, 312-323.

Ridderinkhof,K.R., Forstmann, B.U.,Wylie, S.A., Burle, B., and van den Wildenberg, W.P.M. (2010). Neurocognitive mechanisms of action control: resisting the call of the sirens. Wiley Interdiscip. Rev. Cogn. Science. doi: 10.1002/wcs.99.

Ridderinkhof, K. R., Scheres, A. Oosterlaan, J., and Sergeant, J. (2005). Distribution-analytical techniques in the study of $\mathrm{AD} / \mathrm{HD}$ : delta plot analyses reveal deficits in response suppression that are eliminated by methylphenidate treatment. J. Abnorm. Psychol. $114,197-215$

Ridderinkhof, K. R., Ullsperger, M., Crone, E. A., and Nieuwenhuis, S. (2004a). The role of the medial frontal cortex in cognitive control. Science 306, 443-447.

Ridderinkhof, K. R., van den Wildenberg, W. P. M., Segalowitz, S. J., and Carter, C.S. (2004b). Neurocognitive mechanisms of cognitive control: the role of prefrontal cortex in action selection, response inhibition, performance monitoring, and reward-based learning. Brain Cogn. 56, 129-140.

Ridderinkhof, K. R., van den Wildenberg, W. P. M., Wijnen, J., and Burle, B. (2004c). "Response inhibition in conflict tasks is revealed in delta plots," in Cognitive Neuroscience of Attention, ed. M. Posner (New York: Guilford Press), 369-377.

Roger, C., Bénar, C. G., Vidal, F., Hasbroucq, T., and Burle, B. (2010). Rostral cingulate zone and correct response monitoring: ICA and source localisation evidences for the unicity of correct- and error-negativities. Neuroimage 51, 391-403.

Scheffers, M.K., Coles, M.G.H., Bernstein, P., Gehring, W. J., and Donchin, E. (1996). Event-related brain potentials and error-related processing: an analysis of incorrect responses to go and no-go stimuli. Psychophysiology 33, 42-53.

Schumacher, E. H., Cole, M. W., and D’Esposito, M. (2007). Selection and maintenance of stimulus-response rules during preparation and performance of a spatial choice-reaction task. Brain Res. 1136, 77-87.

Schumacher, E. H., Elston, P. A., and D’Esposito, M. (2003). Neural evidence for representation-specific response selection. J. Cogn. Neurosci. $15,1111-1121$

Simon, J. R. (1967). Ear preference in a simple reaction-time task. J. Exp. Psychol. 75, 49-55.

Simon, J. R. (1990). "The effect of an irrelevant directional cue on human information processing," in StimulusResponse Compatibility: An Integrated Perspective, eds R.W. Proctor and T.G. Reeve (Amsterdam: North-Holland), 31-88.

Smid,H.G.O.M., Mulder, G., and Mulder, L. J. M. (1990). Selective response activation can begin before stimulus recognition is complete: a psychophysiological and error analysis of the continuous flow. Acta Psychol. 74 169-201.

Speckman, P. L., Rouder, J. N., Morey, R. D., and Pratte, M.S. (2008). Delta plots and coherent distribution ordering. Am. Stat. 62, 262-266.

Stins, J.F., Polderman,J.C., Boomsma, D. I, and de Geus, E.J.C. (2007).Conditional accuracy in response interference tasks: evidence from the Eriksen flanker task and the spatial conflict task. Adv. Cogn Psychol. 3, 389-396.

Stroop, J. (1935). Studies of interference in serial verbal reactions. J. Exp. Psychol. $18,643-662$.

Stürmer, B., Leuthold, H., Soetens, E., Schröter, H., and Sommer, W. (2002) Control over location-based response activation in the Simon task: behavioral and electrophysiological evidence. J. Exp. Psychol. Hum. Percept. Perform. 28, 1345-1363.

van den Wildenberg, W. P. M., Burle, B., Vidal, F., van der Molen, M. W. Ridderinkhof, K. R., and Hasbroucq, T. (2010). Mechanisms and dynamics of cortical motor enhibition in the stop-signal paradigm: a TMS study. J. Cogn. Neurosci. 22, 225-239.

van den Wildenberg, W.P.M., van Boxtel, G. J. M., van der Molen, M. W., Bosch, D. A., Speelman, J. D., and Brunia, C. H. M. (2006). Stimulation of the subthalamic region facilitates the selection and inhibition of motor responses in Parkinson's disease. J. Cogn. Neurosci. $18,626-636$

Verbruggen, F., Aron, A. R., Stevens, M A., and Chambers, C. D. (2010). Thet burst stimulation dissociates attention and action updating in human inferior frontal cortex. Proc. Natl. Acad. Sci. U.S.A. 107, 13966-13971.

Vidal, F., Hasbroucq, T., Grapperon, J., and Bonnet, M. (2000). Is the error negativity specific to errors? Biol. Psychol. 51, 109-128.

Wagenmakers, E.-J., Grasman, R. P. P. P., and Molenaar, P. C. M. (2005). On the relation between the mean and the variance of a diffusion model response time distribution. J. Math. Psychol. 49, 195-204.

Wiegand, K., and Wascher, E. (2005). Dynamic aspects of S-R correspondence: evidence for two mechanisms involved in the Simon effect. J. Exp. Psychol. Hum. Percept. Perform. 31 453-464.
Wiegand, K., and Wascher, E. (2007). Response coding in the Simon task. Psychol. Res. 71, 219-233.

Wijnen, J. G., and Ridderinkhof, K. R. (2007). Response inhibition in motor and oculomotor conflict tasks: different mechanisms, different dynamics? Brain Cogn. 63, 260-270.

Wylie, S. A., Ridderinkhof, K. R., Bashore, T. R., and van den Wildenberg, W.P.M. (2010). The effect of Parkinson's disease on the dynamics of online and proactive cognitive control during action selection. J. Cogn. Neurosci. 22, 2058-2073.

Wylie, S. A., Ridderinkhof, K. R., Eckerle, M. K., and Manning, C. A. (2007). Inefficient response inhibition in individuals with mild cognitive impairment. Neuropsychologia 45 1408-1419.

Wylie, S. A., Ridderinkhof, K. R., Elias, W. J., Frysinger, R. C., Bashore, T. R., Downs, K. E., van Wouwe, N. C., and van den Wildenberg, W. P. M. (2010). Subthalamic nucleus stimulation influences expression and suppression of impulsive behavior in Parkinson's disease. Brain. doi: 10.1093/brain/awq239

Wylie, S. A., van den Wildenberg, W. P. M, Ridderinkhof, K. R., Bashore, T. R., Powell, V. D., Manning, C. A., and Wooten, G. F. (2009a). The effect of Parkinson's disease on interference control during action selection. Neuropsychologia 47, 145-157.

Wylie, S. A., van den Wildenberg, W. P. M, Ridderinkhof, K. R., Bashore, T. R., Powell, V. D., Manning, C. A., and Wooten, G. F. (2009b). The effect of speed-accuracy strategy on response interference control in Parkinson's disease. Neuropsychologia 47, 1844-1853.

Conflict of Interest Statement: The authors declare that the research was conducted in the absence of any commercial or financial relationships that could be construed as a potential conflict of interest.

Received: 01 September 2010; accepted: 16 November 2010; published online: 13 December 2010.

Citation: van den Wildenberg WPM, Wylie $S A$, Forstmann BU, Burle B, Hasbroucq T and Ridderinkhof KR (2010) To head or to heed? Beyond the surface of selective action inhibition: a review. Front. Hum. Neurosci. 4:222. doi: 10.3389/fnhum.2010.00222

Copyright $(92010$ van den Wildenberg, Wylie, Forstmann, Burle, Hasbroucq and Ridderinkhof. This is an open-access article subject to an exclusive license agreement between the authors and the Frontiers Research Foundation, which permits unrestricted use, distribution, and reproduction in any medium, provided the original authors and source are credited. 